\title{
The Impact of Ripening on Manganese-Doped ZnSe Nanocrystals
}

\author{
Lijun Zu, David J. Norris, ${ }^{*}{ }^{\dagger}$ Thomas A. Kennedy, ${ }^{\S}$ Steven C. Erwin ${ }^{\ddagger}$ and Alexander L. Efros ${ }^{\dagger}$
}

University of Minnesota, 421 Washington Avenue SE, Minneapolis, MN 55455, and Naval Research Laboratory, 4555 Overlook Avenue SW, Washington, DC 20375

*Corresponding author. E-mail: dnorris@umn.edu

${ }^{\dagger}$ Department of Chemical Engineering and Materials Science, University of Minnesota.

${ }^{\S}$ Electronics Science and Technology Division, Naval Research Laboratory.

$\$$ Materials Science and Technology Division, Naval Research Laboratory.

\section{Supporting Information}

Chemicals. Diethylzinc $\left(\mathrm{ZnEt}_{2}\right)$ was purchased from Strem. Selenium shot was purchased from Alfa Aesar. Tri- $n$-octylphosphine (TOP), hexadecylamine (HDA), and 1-butanol were purchased from Aldrich. Methanol, ethanol, and hexane were purchased from EM Science. Dimethylmanganese $\left(\mathrm{MnMe}_{2}\right)$ was prepared according to a published procedure. ${ }^{\mathrm{S} 1}$ 
Synthesis of ZnSe:Mn nanocrystals. $15 \mathrm{~mL}$ of distilled HDA was degassed under vacuum for 3 hours and then heated to $310^{\circ} \mathrm{C}$ in a reaction flask under dry nitrogen. A precursor mixture of TOP $(4 \mathrm{~mL}), 1 \mathrm{M}$ TOPSe $(0.8 \mathrm{~mL}, 0.8 \mathrm{mmol}), \mathrm{ZnEt}_{2}(82 \mu \mathrm{L}, 0.8 \mathrm{mmol})$, and $0.04 \mathrm{M} \mathrm{MnMe}_{2}$ in a tetrahydrofuran/toluene mixture ${ }^{\mathrm{S} 1}(0.5 \mathrm{~mL}, 0.02 \mathrm{mmol})$ was combined in a $10 \mathrm{~mL}$ syringe inside a nitrogen glove box and rapidly injected into the hot HDA with vigorous stirring. In this reaction, the molar ratio of Se:Zn:Mn is 1:1:0.025 and the initial $\mathrm{Zn}$ precursor concentration in the reaction solution, $[\mathrm{Zn}]^{0}$, is $0.040 \mathrm{M}$. Nanocrystals were grown at temperatures in the range of $260-300^{\circ} \mathrm{C}$ after nucleation. Aliquots (a needle-tip amount) of the reaction solution were removed at regular intervals and diluted with hexane. UV-visible absorption spectra were taken to monitor the growth of the crystallites. Once a desired size was achieved, larger aliquots $(1 \mathrm{~mL})$ of the reaction solution were removed for optical measurements. These samples were precipitated with methanol and redispersed in hexane to get an optical density of around 0.2. The remaining growth solution was heated and this sequence continued until a growth series was obtained.

The data shown in Fig. 1 were obtained using the synthesis described above.

The data shown in Fig. 2 were obtained by using slight modifications of the procedure described above. In particular, the Se:Zn:Mn molar ratios and $[\mathrm{Zn}]^{0}$ were varied. For the synthesis where the Se:Zn:Mn molar ratio was 1:1:0.05 and $[\mathrm{Zn}]^{0}$ was $0.020 \mathrm{M}$, a precursor mixture of TOP $(4 \mathrm{~mL}), 1 \mathrm{M}$ TOPSe $(0.4 \mathrm{~mL}, 0.4 \mathrm{mmol}), \mathrm{ZnEt}_{2}(41 \mu \mathrm{L}, 0.4 \mathrm{mmol})$, and $0.04 \mathrm{M}$ $\mathrm{MnMe}_{2}$ in a tetrahydrofuran/toluene mixture ${ }^{\mathrm{S} 1}(0.5 \mathrm{~mL}, 0.02 \mathrm{mmol})$ was injected into the hot HDA. For the synthesis where the Se:Zn:Mn molar ratio was 1:1:0.05 and $[\mathrm{Zn}]^{0}$ was $0.040 \mathrm{M}$, a precursor mixture of TOP $(4 \mathrm{~mL}), 1 \mathrm{M}$ TOPSe $(0.8 \mathrm{~mL}, 0.8 \mathrm{mmol}), \mathrm{ZnEt}_{2}(82 \mu \mathrm{L}, 0.8 \mathrm{mmol})$, and 
$0.04 \mathrm{M} \mathrm{MnMe}_{2}$ in a tetrahydrofuran/toluene mixture ${ }^{\mathrm{S} 1}(1.0 \mathrm{~mL}, 0.04 \mathrm{mmol})$ was injected. For the synthesis where the Se:Zn:Mn molar ratio was $1: 1: 0.05$ and $[\mathrm{Zn}]^{0}$ was $0.074 \mathrm{M}$, a precursor mixture of TOP $(4 \mathrm{~mL}), 1 \mathrm{M}$ TOPSe $(1.6 \mathrm{~mL}, 0.4 \mathrm{mmol}), \mathrm{ZnEt}_{2}(164 \mu \mathrm{L}, 1.6 \mathrm{mmol})$, and $0.04 \mathrm{M}$ $\mathrm{MnMe}_{2}$ in a tetrahydrofuran/toluene mixture ${ }^{\mathrm{S} 1}(2.0 \mathrm{~mL}, 0.08 \mathrm{mmol})$ was injected into the hot HDA. For the synthesis where the Se:Zn:Mn molar ratio was 4:1:0.05 and $[\mathrm{Zn}]^{0}$ was $0.016 \mathrm{M}$, a precursor mixture of TOP $(3.7 \mathrm{~mL}), 1 \mathrm{M}$ TOPSe $\left(1.28 \mathrm{~mL}, 1.28 \mathrm{mmol}^{2}, \mathrm{ZnEt}_{2}(32.8 \mu \mathrm{L}\right.$, $0.32 \mathrm{mmol})$, and $0.04 \mathrm{M} \mathrm{MnMe}_{2}$ in a tetrahydrofuran/toluene mixture ${ }^{\mathrm{S} 1}(0.4 \mathrm{~mL}, 0.016 \mathrm{mmol})$ was injected. For the synthesis where the Se:Zn:Mn molar ratio was 1:2:0.10 and $[\mathrm{Zn}]^{0}$ was $0.053 \mathrm{M}$, a precursor mixture of TOP $(4 \mathrm{~mL}), 1 \mathrm{M}$ TOPSe $(0.53 \mathrm{~mL}, 0.53 \mathrm{mmol}), \mathrm{ZnEt}_{2}(109 \mu \mathrm{L}$, $1.06 \mathrm{mmol})$, and $0.04 \mathrm{M} \mathrm{MnMe}_{2}$ in a tetrahydrofuran/toluene mixture ${ }^{\mathrm{S} 1}(1.33 \mathrm{~mL}, 0.053 \mathrm{mmol})$ was injected.

The data shown in Fig. 3 were obtained using a synthesis where the Se:Zn:Mn molar ratio was 4:1:0.05 and $[\mathrm{Zn}]^{0}$ was $0.016 \mathrm{M}$ as described above. More reactants were then added dropwise at $270^{\circ} \mathrm{C}$ starting at 58 and 88 minutes, respectively. Each of these secondary precursor solutions contained $2 \mathrm{~mL}$ of TOP, $0.64 \mathrm{~mL}$ of $1 \mathrm{M}$ TOPSe, $16.4 \mu \mathrm{L}$ of $\mathrm{ZnEt}_{2}$, and $0.2 \mathrm{~mL}$ of $0.04 \mathrm{M} \mathrm{MnMe}_{2}$ in a tetrahydrofuran/toluene mixture ${ }^{\mathrm{S} 1}$ and was added within 20 minutes.

The data shown in Figs. $4 \mathrm{a}$ and $4 \mathrm{~b}$ were obtained by repeating the reactions in Fig. $2 \mathrm{~b}$ with two slow additions of the precursors. The secondary precursor solution contained half the amount of the initial injection solution and was added dropwise before the intensity ratio reached the maximum. The time for this addition was obtained from Fig $2 \mathrm{~b}$. The data shown in Figs. 4c and $4 \mathrm{~d}$ were obtained with the same procedure, except the $\mathrm{Zn}: \mathrm{Mn}$ molar ratio was 1:0.025. 
The data shown in Fig. 6 were obtained by repeating the reaction shown in Fig. 3. However, additional precursors were added only once. For each aliquot collected, the nanocrystals were precipitated and treated with pyridine twice to reduce surface $\mathrm{Mn}^{\mathrm{S} 2}$ Each sample was then divided for ICP and EPR analysis as described below. From absorption data, the average size of the nanocrystals in each aliquot could be determined. This was then used to extract the average number of $\mathrm{Zn}$ atoms per particle. By measuring the total $\mathrm{Zn}: \mathrm{Mn}$ ratio with ICP and then correcting for the fact that some of the Mn were on the surface, the average number of Mn per nanocrystal was obtained.

Photoluminescence Measurements. Photoluminescence spectra were collected with a Spex Fluorolog-2 spectrofluorometer, equipped with two monochromators (double-grating, $0.22 \mathrm{~m}$, SPEX 1680) and a 450W xenon lamp as the excitation source. All photoluminescence spectra were corrected for the spectral response of the emission monochromator and the photomultiplier tube. For each aliquot, $1 \mathrm{~mL}$ of the reaction solution was removed, precipitated with methanol, redispersed in hexane to get an optical density of around 0.2 , and then the PL spectrum was recorded.

The intensity ratio, $I_{M n} / I_{Z n S e}$, was extracted from such spectra using the following equation

$$
\frac{I_{M n}}{I_{\text {ZnSe }}}=\frac{\int_{M n} I_{M n}(\lambda) d \lambda}{\int_{\text {ZnSe }} I_{\text {ZnSe }}(\lambda) d \lambda}
$$

where the numerator is an integral over the Mn emission band and the denominator is an integral over the exciton emission band; $I_{M n}(\lambda)=A \cdot \frac{R_{M n}(\lambda)}{\lambda}, I_{Z n S e}(\lambda)=A \cdot \frac{R_{Z n S e}(\lambda)}{\lambda}, A$ is a constant, $\lambda$ is the optical wavelength, and $R_{M n}(\lambda)$ and $R_{Z n S e}(\lambda)$ are the number of photons per second detected in the Mn emission band and in the exciton emission band, respectively. 
Electron Paramagnetic Resonance (EPR). For the aliquots analyzed in Fig. 6, half of the nanocrystals were precipitated and cast in polylaurylmethacrylate films. Q-band (35 GHz) EPR spectra were then collected on a Varian E109Q spectrometer at room temperature. The microwave frequency was chosen to reduce line broadening that arises from inhomogeneities in the crystal field at the Mn sites.

A typical EPR spectrum for these samples after two pyridine exchanges is shown in Fig. S1. The spectra exhibit two hyperfine coupling constants, one for the incorporated Mn and one for the Mn that is on the surface. The relative amount of surface Mn can be approximated by taking the ratio of the signal at $1.27 \mathrm{~T}$, where the surface Mn dominates, and at $1.227 \mathrm{~T}$, where the incorporated Mn dominates. After two pyridine exchanges, we find that $\sim 40 \%$ of the total $\mathrm{Mn}$ in the sample remains on the surface.

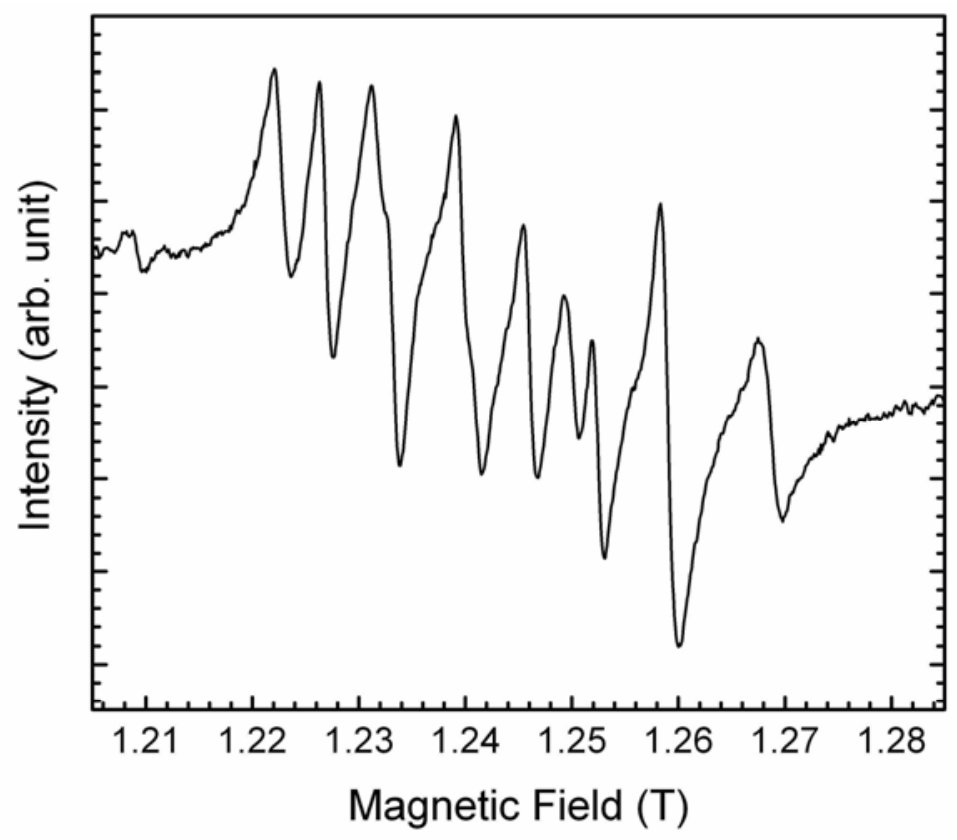

Fig. S1 A typical EPR spectrum for the Mn-doped ZnSe nanocrystals analyzed in Fig. 6. The nanocrystals were measured at room temperature after two pyridine exchanges. The spectrum shows two sets of hyperfine splittings with coupling constants $61.6 \times 10^{-4} \mathrm{~cm}^{-1}$ for the incorporated $\mathrm{Mn}$ and $89 \times 10^{-4} \mathrm{~cm}^{-1}$ for the surface bound Mn. 
Inductively Coupled Plasma Atomic Emission Spectroscopy (ICP). The Zn:Mn ratio was determined by ICP (Perkin-Elmer Optima 3000DV). Prior to analysis, the ZnSe:Mn nanocrystals were dissolved in aqua regia.

Optical Data. Figures S2, S3, S4, S5, and S6 below present absorption and photoluminescence data as a function of growth for the same samples shown in Figs. 3 and 4.

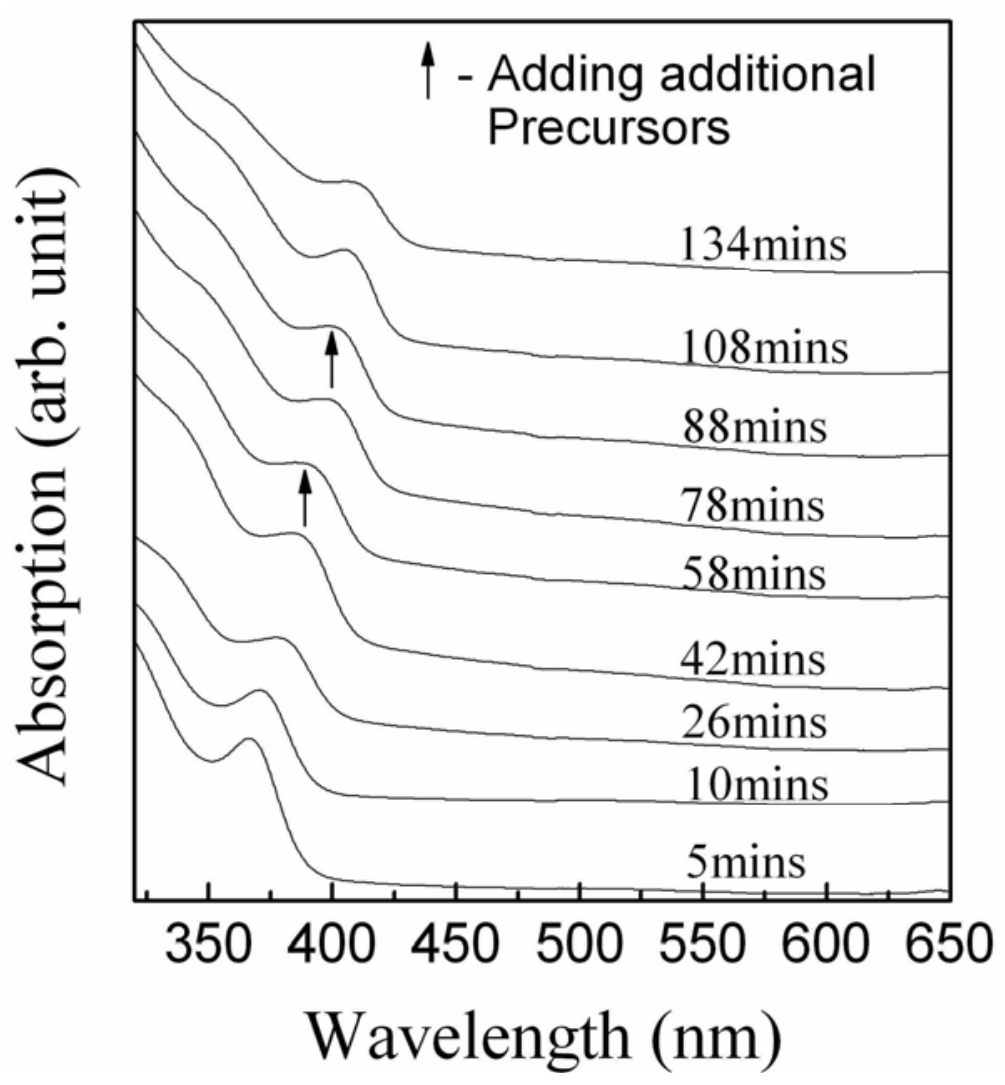

Fig. S2 Absorption spectra for ZnSe:Mn nanocrystals as a function of growth time. These samples are the same as shown in Fig. 3. More reactants were added after the onset of ripening at the positions of the vertical arrows, as described in the text. 


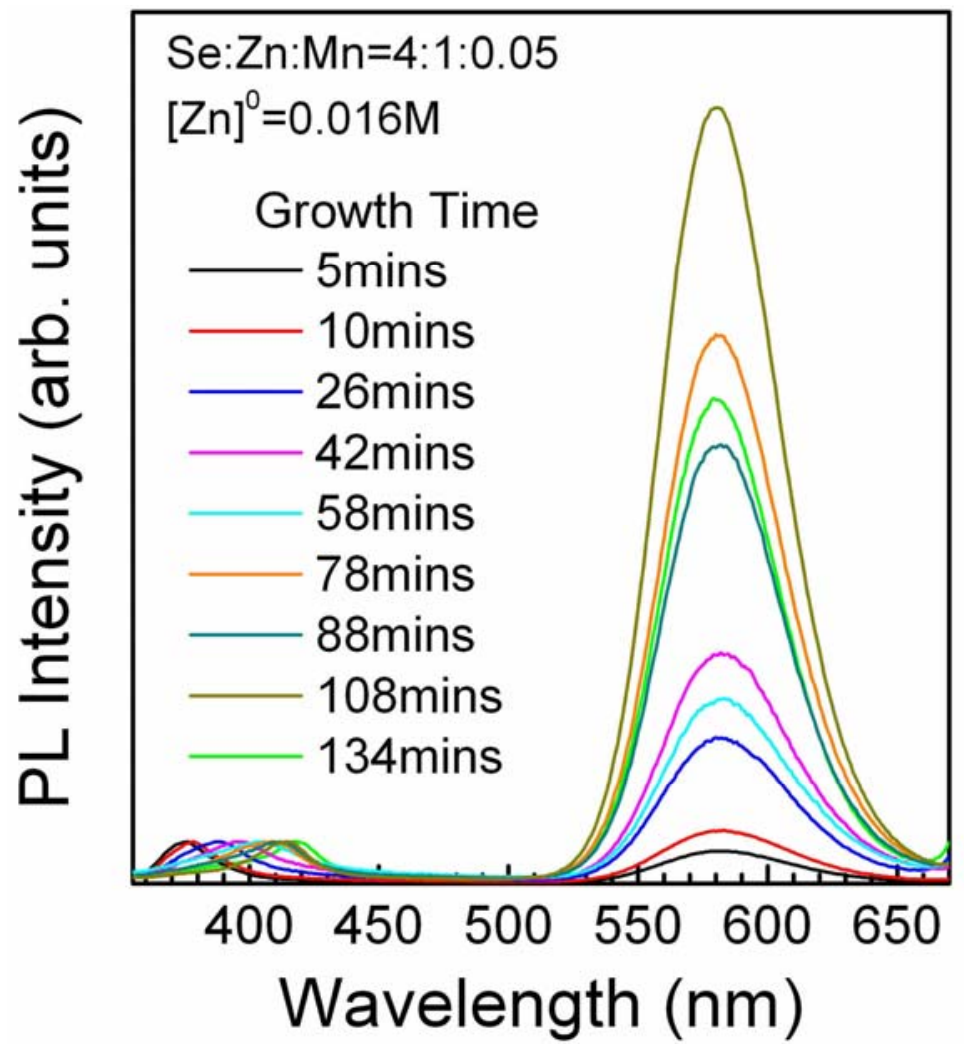

Fig. S3 Photoluminescence spectra for Mn-doped $\mathrm{ZnSe}$ nanocrystals as a function of growth time (in minutes). All of the spectra are normalized to the magnitude of the exciton emission band. The intensity ratio, $I_{M n} / I_{Z n S}$, versus growth time for these data are shown in Fig. 3. 


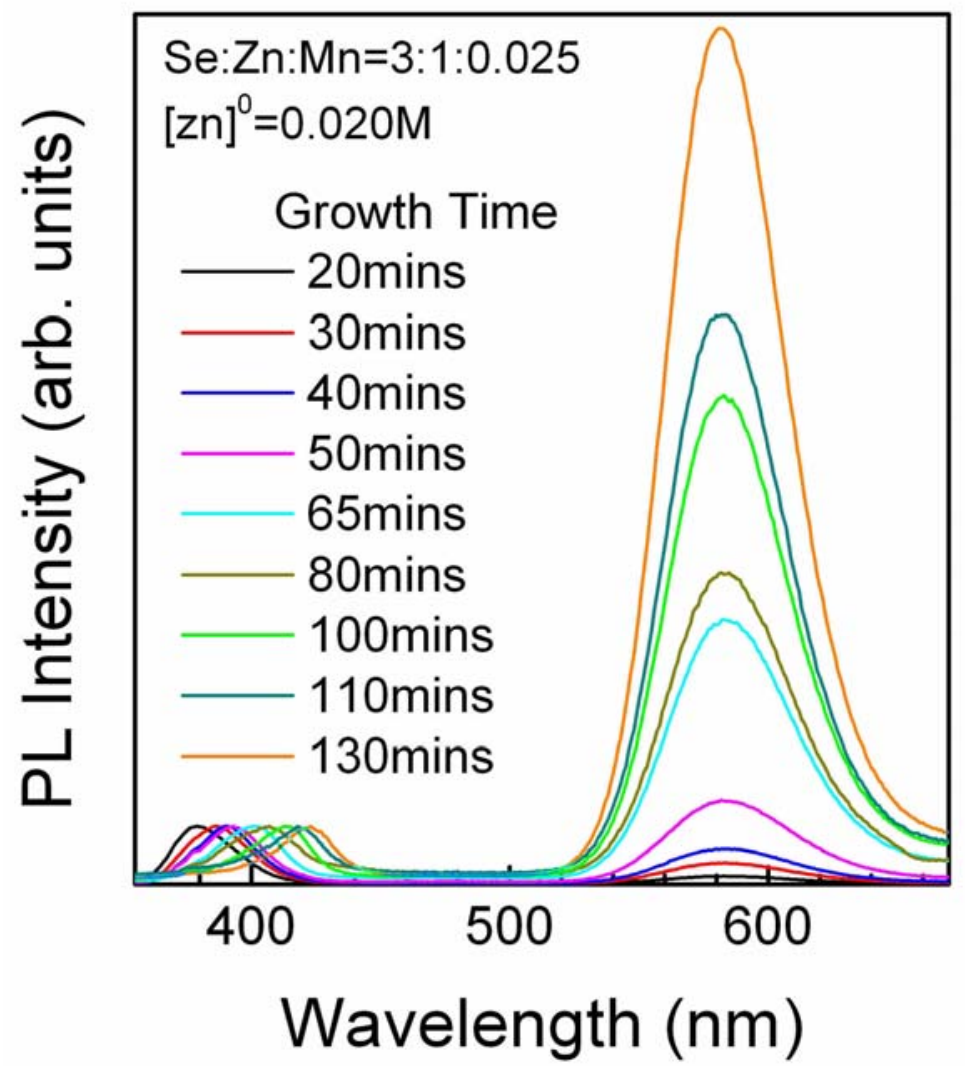

Fig. S4 Photoluminescence spectra for Mn-doped $\mathrm{ZnSe}$ nanocrystals as a function of growth time (in minutes). All of the spectra are normalized to the magnitude of the exciton emission band. The intensity ratio, $I_{M n} / I_{Z n S e}$, versus growth time for these data are shown in Fig. 4. 


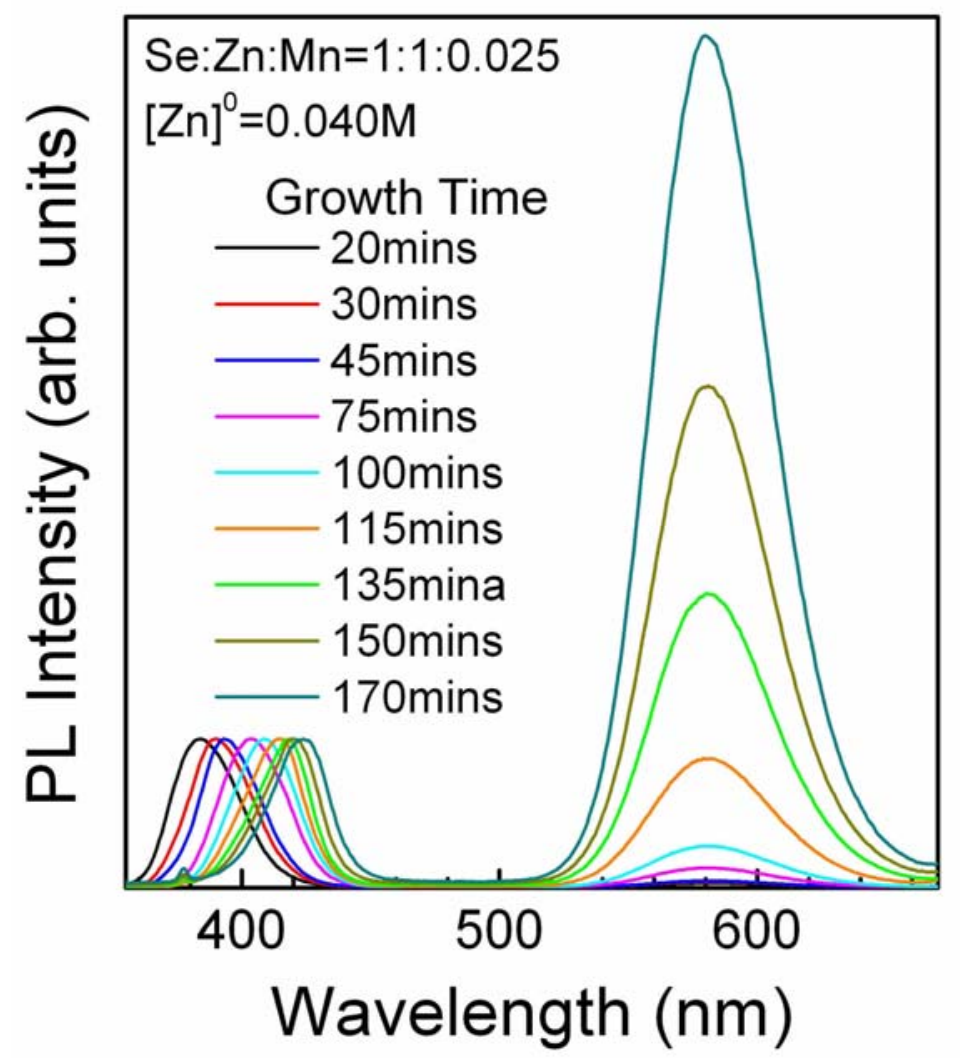

Fig. S5 Photoluminescence spectra for Mn-doped ZnSe nanocrystals as a function of growth time (in minutes). All of the spectra are normalized to the magnitude of the exciton emission band. The intensity ratio, $I_{M n} / I_{Z n S e}$, versus growth time for these data are shown in Fig. 4. 


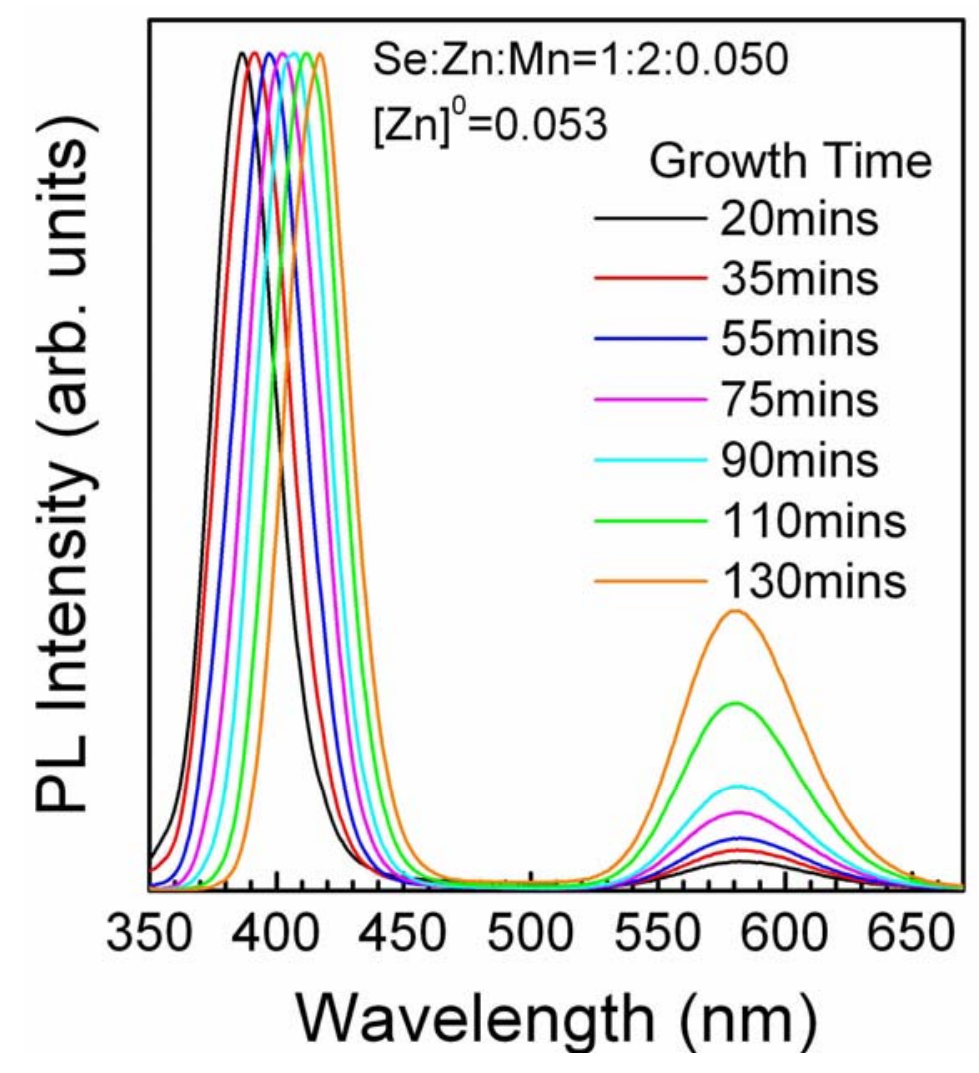

Fig. S6 Photoluminescence spectra for Mn-doped $\mathrm{ZnSe}$ nanocrystals as a function of growth time (in minutes). All of the spectra are normalized to the magnitude of the exciton emission band. The intensity ratio, $I_{M n} / I_{Z n S e}$, versus growth time for these data are shown in Fig. 4. 
Theoretical Proof of Equation 4. Previously, ${ }^{\mathrm{S} 3}$ we showed that the ensemble-averaged intensities for the Mn and the exciton emissions are

$$
\begin{aligned}
& \left\langle I_{M n}\right\rangle=Q_{M n} R E_{M n} \sum_{N} \frac{N P_{\bar{N}}(N)}{\tau_{c} / \tau+N} \\
& \left\langle I_{Z n S e}\right\rangle=R \frac{\tau_{c}}{\tau_{r}} E_{Z n S e} \sum_{N} \frac{P_{\bar{N}}(N)}{\tau_{c} / \tau+N},
\end{aligned}
$$

where $R$ is the absorption rate of the nanocrystals, $\tau^{-1}$ is the total (radiative plus non-radiative) exciton relaxation rate of undoped nanocrystals, $E_{M n}$ and $E_{Z n S e}$ are the photon energies for Mn and exciton emission, respectively, and $P_{\bar{N}}(N)$ is the Poissonian distribution, i.e.,

$$
P_{\bar{N}}(N)=\sum_{N=0}^{\infty} \bar{N}^{N} \frac{\exp (-\bar{N})}{N !}
$$

If we first assume that $\bar{N} \gg 1\left(\right.$ such that $\left.P_{\bar{N}}(0) \approx 0\right)$, then

$$
\begin{aligned}
& \left\langle I_{M n}\right\rangle=Q_{M n} R E_{M n} \sum_{N>0} \frac{N P_{\bar{N}}(N)}{\tau_{c} / \tau+N} \\
& \left\langle I_{Z n S e}\right\rangle=R \frac{\tau_{c}}{\tau_{r}} E_{Z n S e} \sum_{N>0} \frac{P_{\bar{N}}(N)}{\tau_{c} / \tau+N}
\end{aligned}
$$

Second, we assume that $\tau_{c} / \tau \ll 1$. (According to the Supplementary Methods section in Ref. S3, $\tau_{c} / \tau=0.3$.) Thus, eqs S-4 simplify to

$$
\begin{gathered}
\left\langle I_{M n}\right\rangle=Q_{M n} R E_{M n} \sum_{N>0} \frac{N P_{\bar{N}}(N)}{N}=Q_{M n} R E_{M n} \sum_{N>0} P_{\bar{N}}(N)=Q_{M n} R E_{M n} \\
\left\langle I_{\text {ZnSe }}\right\rangle=R \frac{\tau_{c}}{\tau_{r}} E_{\text {ZnSe }} \sum_{N>0} \frac{P_{\bar{N}}(N)}{N}=R \frac{\tau_{c}}{\tau_{r}} E_{\text {ZnSe }} \sum_{N>0} N^{-1} P_{\bar{N}}(N)=R \frac{\tau_{c}}{\tau_{r}} E_{\text {ZnSe }} \overline{N^{-1}}
\end{gathered}
$$

The intensity ratio is then

$$
\frac{\left\langle I_{M n}\right\rangle}{\left\langle I_{Z n S e}\right\rangle}=Q_{M n} \frac{\tau_{r}}{\tau_{c}} \frac{E_{M n}}{E_{Z n S e}}\left(\overline{N^{-1}}\right)^{-1}=Q_{M n} \frac{\tau_{r}}{\tau_{c}} \frac{\lambda_{\text {ZnSe }}}{\lambda_{M n}}\left(\overline{N^{-1}}\right)^{-1}=C\left(\overline{N^{-1}}\right)^{-1}
$$

The question then turns to whether

$$
\left(\overline{N^{-1}}\right)^{-1}=\bar{N} \quad ?
$$

For the Poissonian distribution, this is indeed the case for $\bar{N} \gg 1$, as can be shown by starting with 


$$
\left(\overline{N^{-1}}\right)^{-1}=1 / \sum_{N=0}^{\infty} N^{-1} P_{\bar{N}}(N)=1 / \sum_{N=0}^{\infty} N^{-1} \bar{N}^{N} \frac{\exp (-\bar{N})}{N !}=1 / \exp (-\bar{N}) \sum_{N=0}^{\infty} \frac{\bar{N}^{N}}{(N+1) !}
$$

Then, let $M=N+1$ and $\bar{M}=\bar{N}$ such that

$$
\left(\overline{N^{-1}}\right)^{-1}=1 / \exp (-\bar{M}) \sum_{M=1}^{\infty} \frac{\bar{M}^{M-1}}{M !}=\bar{M} / \exp (-\bar{M}) \sum_{M=1}^{\infty} \frac{\bar{M}^{M}}{M !}=\bar{M} / \exp (-\bar{M})\left[-1+\sum_{M=0}^{\infty} \frac{\bar{M}^{M}}{M !}\right]
$$

or

$$
\left(\overline{N^{-1}}\right)^{-1}=\bar{M} /\left[-\exp (-\bar{M})+\sum_{M=0}^{\infty} \frac{\bar{M}^{M} \exp (-\bar{M})}{M !}\right]=\bar{N} /\left[-\exp (-\bar{N})+\sum_{N=0}^{\infty} \frac{\bar{N}^{N} \exp (-\bar{N})}{N !}\right]
$$

If $\bar{N} \gg 1$, then

$$
\left(\overline{N^{-1}}\right)^{-1} \approx \bar{N} /\left[\sum_{N=0}^{\infty} \frac{\bar{N}^{N} \exp (-\bar{N})}{N !}\right]=\bar{N} /\left[\sum_{N=0}^{\infty} P_{\bar{N}}(N)\right]=\bar{N}
$$

Therefore

$$
\frac{\left\langle I_{M n}\right\rangle}{\left\langle I_{\text {ZnSe }}\right\rangle}=C\left(\overline{N^{-1}}\right)^{-1}=C \bar{N}
$$

\section{References:}

(S1) Norris, D. J.; Yao, N.; Charnock, F. T.; Kennedy, T. A. Nano Lett. 2001, 1, 3.

(S2) Mikulec, F. V.; Kuno, M.; Bennati, M.; Hall, D. A.; Griffin, R. G.; Bawendi, M. G. J. Am. Chem. Soc. 2000, 122, 2532.

(S3) Erwin, S. C.; Zu, L.; Haftel, M. I.; Efros, A. L.; Kennedy, T. A.; Norris, D. J. Nature 2005, 436, 91. 